\title{
HIPERPIGMENTACIÓN DE LAS PAPILAS FUNGIFORMES. REPORTE DE UN CASO CLÍNICO
}

\author{
Fungiform papillae hyperpigmentation. Clinical case report
}

\author{
Priscila Fernández M. ${ }^{\text {, }}$ María del Carmen Pariona M. ${ }^{\text {, }}$, María Gabriela Patiño R. ${ }^{2}$ \\ ${ }^{1}$ Docentes de la Carrera de Odontología- Universidad Católica de Cuenca. \\ ${ }^{2}$ Clínico, estomatólogo, oncólogo y cirujano traumatólogo Maxilo Facial de la Clínica Médica Del Sur
}

DOI: https://doi.org/10.31984/oactiva.v6i3.422

\begin{abstract}
Resumen
Las papilas linguales, especialmente las fungiformes pueden presentar hiperpigmentación en pacientes de tez oscura. Se caracteriza por la pigmentación localizada y circunscrita de las papilas. Siendo su patogénesis desconocida, resulta importante el diagnóstico clínico y de ser necesario el uso de métodos auxiliares como la dermatoscopia. Se presenta el caso de un paciente de 8 años de edad que presentaba múltiples máculas hipercrómicas en la lengua.
\end{abstract}

Palabras Claves: Trastornos de Pigmentaciones, papilas gustativas

\begin{abstract}
Lingual papillae, especially the fungiform ones, may present hyperpigmentation in dark-skinned patients. This disorder is known for its localized and circumscribed papillae pigmentation. The pathogenesis is unknown; therefore, the clinical diagnosis becomes essential, as well as the use of auxiliary methods such as dermoscopy, if necessary. This paper presents the case of an 8-year-old patient with numerous hyperchromic macules on the tongue.
\end{abstract}

Keywords: Pigmentation disorders, taste buds

\section{Introducción}

En la lengua encontramos 4 tipos diferentes de papilas: caliciformes o circunvaladas, fungiformes, filiformes y foliadas. ${ }^{1,2}$ Se describen como receptores sensitivos especializados en el sentido del gusto que, ubicadas en diferentes zonas de la lengua detectan diferentes sabores y estímulos. Las papilas fungiformes están específicamente localizadas en la punta y zonas laterales de la lengua, presentan forma de hongo, de ahí su nombre; se definen como elevaciones epiteliales cubriendo un núcleo de tejido conectivo. $^{1,2,3}$
Las papilas fungiformes pigmentadas de la lengua o PFPT por su nombre en inglés (Pigmented fungiform papillae of the tongue), fueron reportadas inicialmente por Leonard en 1905, que las describió como la hiperpigmentación discreta de la superficie de las papilas fungiformes presente especialmente en pacientes negros; posteriormente fue reportada con menos frecuencia en individuos asiáticos, del Medio Oriente y mujeres hindús. $3,4,5$ 
En el estudio de prevalencia realizado por Holzwanger et al, en una muestra de 300 individuos identificaron que el $30 \%$ de mujeres y el $25 \%$ de hombres de raza negra y un individuo blanco presentaban algún tipo de hiperpigmentación en la lengua. ${ }^{3}$

Se la describe como a una condición asintomática no progresiva que en la mayoría de los casos no se encuentra relacionada con ninguna condición sistémica, cuya edad de aparición es la segunda o tercera década de vida y siendo muy poco frecuente en la niñez. ${ }^{2}$

Se han identificado tres tipos clínicos: (I) máculas hiperpigmentadas bien definidas que involucran todas las papilas fungiformes desde la punta hasta los bordes laterales de la lengua. (II) hiperpigmtenaciones que involucran de tres a siete papilas fungiformas, distribuidas de forma aleatoria en la superficie de la lengua, (III) hiperpigmentaciones de todas las papilas de la superficie dorsal de la lengua., ${ }^{4,6}$

La literatura refiere que el PFPT al ser una condición benigna no requiere exámenes auxiliares complementarios invasivos recomendándose la dermatocospia para confirmar el diagnóstico clínico en la cual se observará el aspecto característico de pétalos de rosa. ${ }^{7}$

Una vez establecido el diagnóstico no es necesario ningún tratamiento. ${ }^{7}$

\section{Reporte del caso}

El siguiente reporte de caso, corresponde a un habitante de Sayausi, en Cuenca-Ecuador perteneciente a la provincia del Azuay, el niño pertenece al grupo de pacientes que conforman el proyecto UDIPSAI, programa de labor social que realiza la Universidad Católica de Cuenca-Ecuador.

Paciente masculino de 8 años de edad, a la exploración clínica presenta lesiones de caries en estado activo e inactivo.

Se evidencia la presencia de pigmentaciones a nivel de cara dorsal y bordes laterales de la lengua; no se observan pigmentaciones en labios, mucosa yugal, uñas ni conjuntiva ocular; el representante del paciente indica que sus alimentos son cocidos en fogón de leña, y que su alimentación se basa principalmente en granos y carbohidratos.

El paciente no manifiesta signos clínicos de sangrado o de dolor, relacionados con las pigmentaciones observadas.

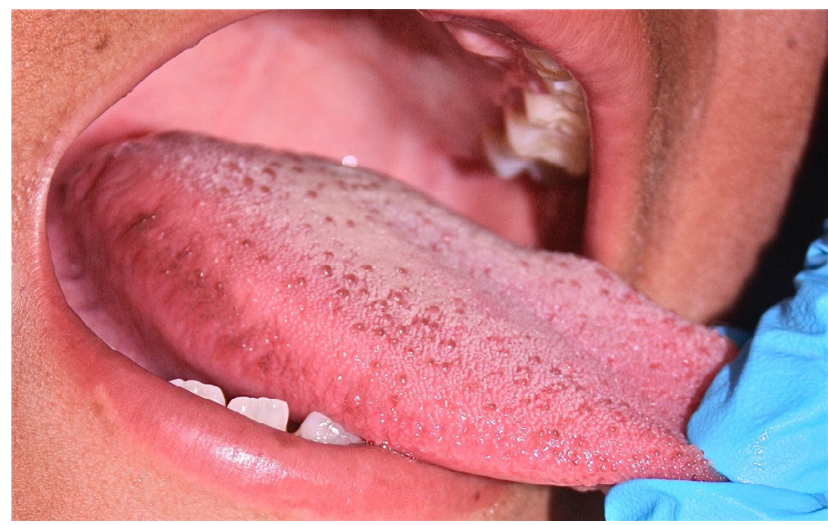

Fig 1. Pigmentaciones visibles borde lateral derecho de la lengua

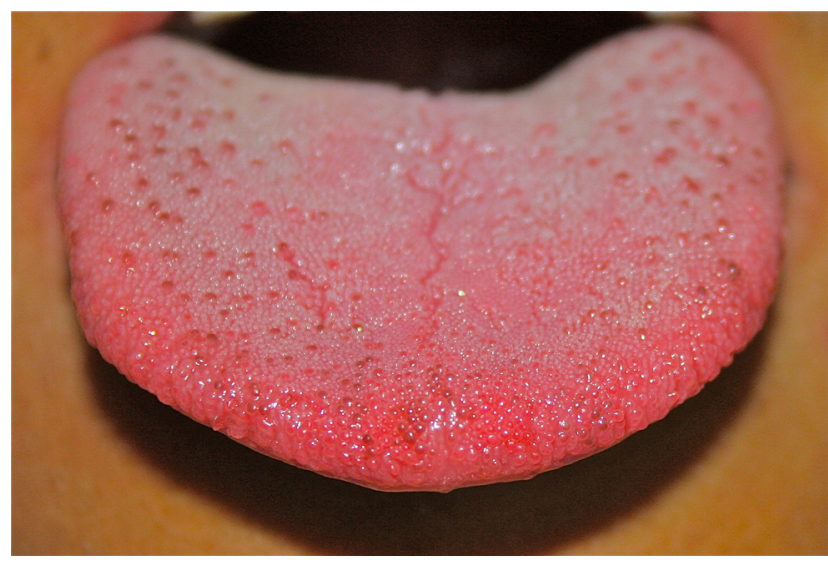

Fig 2. Pigmentaciones visibles en la punta de la lengua.

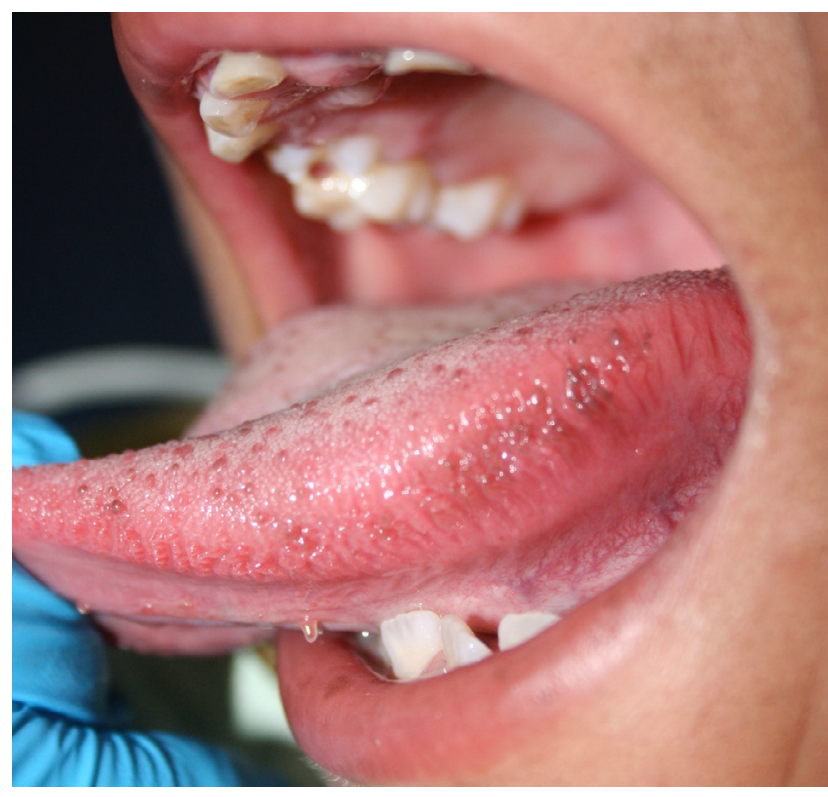

Fig 3. Pigmentaciones visibles en el borde lateral izquierdo de la lengua

Sistémicamente el paciente se encuentra sano. 


\section{Discusión}

Muchas lesiones patológicas se pueden ver y presentar con idénticas o similares características entre sí. Por lo que resulta indispensable la interconsulta con profesionales del área, así como la utilización de exámenes complementarios que permitan realizar un correcto diagnóstico diferencial de ser necesario.

Mukamal et al 2012 refiere que la pigmentación de las papilas, no es una característica inusual, es una variación benigna de la hiperpigmentación oral, presente en adultos y niños con un fototipo de piel alto, donde existen pigmentaciones activas, en donde deberían de estar inactivas. ${ }^{6}$

Existe el reporte de 6 casos clínicos dehiperpigmentación macular mucocutánea benigna, en los que se observa pigmentaciones en mucosa bucal, labios, uñas y conjuntiva. Los autores refieren que estas hiperpigmentaciones son características del síndrome de Laugier-Hunzinker, con el cual debemos hacer un diagnóstico diferencial ${ }^{7}$, el cual se puede establecer fácilmente pues según la literatura, las PFPT se observan en individuos de fototipo de piel alto (oscuro) mientras que el de Laugier-Hunzinker, se presenta en individuos caucásicos. ${ }^{8,9}$

De existir la duda, es necesario realizar la dermatoscopía, en la cual la PFPT se observa como proyecciones con bordes pigmentados que se intercalan con vasos dicotomizados, originados en su base dándole un aspecto similar a los pétalos de rosa, se sugiere este examen específico pues no es invasivo especialmente en caso de niños. ${ }^{6,7}$

\section{Conclusión}

En este reporte, se evidencia la importancia de realizar una historia clínica y una exploración intra y extraoral minuciosa para poder realizar un correcto diagnóstico presuntivo clínico que nos oriente a buscar el tratamiento ideal para la o las patologías, o de ser el caso que el Odontólogo sea quien remita adecuada y responsablemente a la especialidad odontológica o médica que pueda brindar el tratamiento si este no está en nuestras manos.

Concluimos, por el análisis de la anamnesis además de las características clínicas observadas en la exploración física, que estamos frente a una PFPT, la misma que es benigna y no requiere exámenes complementarios invasivos como la biopsia, ni está indicado ningún tratamiento, como lo documenta la literatura revisada.

\section{Bibliografía}

1. Ross MH, Pawlina W. Digestive system I: oral cavity and associated structures. In: Ross MH, Pawlina W. Histology: A Text and Atlas, With Correlated Cell and Molecular Biology. 6th ed. Baltimore, MD: Lippincott Williams \& Wilkins; 2010:526-56

2. Chessa, M. , Patrizi, A., Sechi, A. , Virdi, A. , Leuzzi, M. and Neri, I. (2018), Pigmented fungiform lingual papillae: dermoscopic and clinical features. J Eur Acad Dermatol Venereol, 32: 935-939.

3. Smogorzewski, J.M; Armstrong, P; Young, L. Pigmented Fungiform Papillae of the Tongue in an Indian Male. Cutis. 2019 March;103(3):E16-E17

4. Holzwanger JM, Rudolph RI, Heaton CL. Pigmented fungiform papillae of the tongue: a common variant of oral pigmentation. Int J Dermatol. 1974;13:403-408.

5. Millington GW, Shah SN. A case of pigmented fungiform lingual papillae in an Indian woman. J Eur Acad Dermatol Venereol. 2007;21:705.

6. Luana V. Mukamal, P.; ORMIGA, M.; Ramos-E, S. Dermoscopy of the pigmented fungiform papillae of the tongue.Journal of Dermatology 2012; 39: 397-399.

7. Fajer Al-Fagaan,Bobby Joseph.A Case of Pigmented Fungiform Papillae of the Tongue in a Middle Eastern Woman.Med Princ Pract 2014;23:167-169

8. Urbina F, Sudy E. Pigmented fungiform papillae of the tongue in Laugier disease or Laugier-Hunziker syndrome. Actas Dermosifiliogr. 2013;104:173-174.

9. Díaz-Franco, M.A; Mancheno-Valencia, A.; Vega-Memije, M.E; Mosqueda, T. Síndrome de Laugier-Hunziker: reporte de seis casos e importancia del diagnóstico diferencial. Revista de Ciencias Clínicas. Área de ciencias clínicas. Ciencias Clínicas 2014;15(2):55-61.

Recibido: 28 de noviembre de 2019

Aceptado: 01 de agosto de 2021 
